\title{
Colonic Intramucosal Cancer in the Interposed Colon Treated with Endoscopic Mucosal Resection: A Case Report and Review of Literature
}

\author{
Seung-Ho Baek ${ }^{1}$, Jang-Ho Lee ${ }^{1}$, Dong Ryeol Yoo ${ }^{1}$, Hye Yeong Kim¹, Meihua Jin ${ }^{1}$, Ah-reum Jang ${ }^{1}$, Dong-Hoon Yang ${ }^{2}$ and \\ Jeong-Sik Byeon ${ }^{2}$ \\ Department of ${ }^{1}$ Internal Medicine, ${ }^{2}$ Gastroenterology, Asan Medical Center, University of Ulsan College of Medicine, Seoul, Korea
}

Colon interposition is a surgical procedure used for maintenance of luminal conduit after esophagectomy. Although epithelial neoplasia, such as adenoma and adenocarcinoma, may develop in the interposed colon, there are only few case reports on the condition. Due to the rarity of this condition, there is no definite consensus on recommending screening endoscopy for the early detection of neoplasia in the interposed colons. Here, we report a case of intramucosal adenocarcinoma in an interposed colon. Initial endoscopic resection for this tumor failed to accomplish complete resection. A subsequent endoscopic resection was performed 1 month later and complete resection was achieved. Based on our experience and recommendation on screening endoscopy for gastric cancer in Korea, we suggest that regular screening esophagogastroduodenoscopies should be performed following esophagectomy to detect early neoplasia in the stomach and interposed colon and avoid adverse results induced by delayed detection. Clin Endosc 2019;52:377-381

Key Words: Colon interposition; Adenocarcinoma; Endoscopic resection; Screening endoscopy

\section{INTRODUCTION}

Colon interposition is a surgical procedure for reconstructing the esophageal conduit after esophagectomy, especially in situations in which the stomach cannot be pulled upwards to the oral side. First introduced by Kelling and Vulliet in 1911, colon interposition has been applied to benign conditions, such as esophageal stricture and esophageal cancer. ${ }^{1}$ Briel et al. conducted a study on 393 patients who underwent esophagectomy and reported that the rates of short-term complications, such as conduit ischemia, anastomosis leak, and stricture, after colon interposition were $7.4 \%, 6.1 \%$, and $8.7 \%$, respectively.

Received: July 24, 2018 Revised: January 17, 2019

Accepted: January 30, 2019

Correspondence: Jeong-Sik Byeon

Department of Gastroenterology, Asan Medical Center, University of Ulsan College of Medicine, 88 Olympic-ro 43-gil, Songpa-gu, Seoul 05505, Korea

Tel: +82-2-3010-3905, Fax: +82-2-485-5782, E-mail: jsbyeon@amc.seoul.kr ORCID: https://orcid.org/0000-0002-9793-6379

(cc) This is an Open Access article distributed under the terms of the Creative Commons Attribution Non-Commercial License (http://creativecommons.org/ licenses/by-nc/3.0) which permits unrestricted non-commercial use, distribution, and reproduction in any medium, provided the original work is properly cited.
Importantly, adenoma and adenocarcinoma may develop in interposed colons during a long-term follow-up period; however, only few reports addressed long-term outcomes such as adenoma and adenocarcinoma in interposed colon grafts. Here, we report a case of intramucosal adenocarcinoma in an interposed colon, which was treated by repeated endoscopic resection. Based on our experience as well as literature reviews, we suggest a strategy for appropriate screening and surveillance endoscopies for neoplasia in interposed colons.

\section{CASE REPORT}

A 68 year old female visited the gastroenterology department at our center due to abnormal findings on an esophagogastroduodenoscopy carried out at an outside hospital. The patient had undergone an esophagectomy with right colon interposition for lye-induced corrosive esophagitis 40 years ago. Five years following the esophagectomy, the patient underwent adhesiolysis and small bowel segmental resection with anastomosis due to mechanical ileus resulting from in- 
tra-abdominal adhesions. Since then, no follow-up endoscopy or fecal occult blood test had been performed.

Recently, the patient experienced mild dysphagia and visited an outside hospital; a 20-mm sized non-pedunculated polyp at a distal portion of the interposed colon was detected on esophagogastroduodenoscopy, and endoscopic mucosal resection was attempted. However, en bloc resection could not be achieved due to technical difficulty; therefore, piecemeal resection was performed instead (Fig. 1). Consequently, complete resection was not achieved, and part of the lesion remained. Histological examination of the resected specimen showed a 4-mm sized moderately differentiated adenocarcinoma in the background of a villotubular adenoma. The adenocarcinoma component was confined to the lamina propria (i.e., intramucosal cancer) (Fig. 2). The resection margin was positive due to incomplete piecemeal resection. Per-anal colonoscopy was also performed to the native colon. Three polyps of $5 \mathrm{~mm}$ or less in size were removed by endoscopic polypectomy. Histopathology findings showed tubular adenoma. The patient was referred to the gastroenterology department of our center for management of the incompletely resected tumor at the interposed colon.

She had no past medical history, family history, or social history. Initial vital signs were within normal range: blood pressure of 135/86 $\mathrm{mm} \mathrm{Hg}$, respiration rate of $18 / \mathrm{min}$, pulse rate of $68 / \mathrm{min}$, and blood temperature of $36.7^{\circ} \mathrm{C}$. Her body weight and height were $54.6 \mathrm{~kg}$ and $152 \mathrm{~cm}$, respectively. Laboratory findings were within the normal range.

Esophagogastroduodenoscopy was carried out with a gastroscope (GIF HQ 290; Olympus Co., Tokyo, Japan). The proximal anastomosis site between the esophagus and the interposed colon was found $18 \mathrm{~cm}$ from the upper incisor, and the distal anastomosis site between the interposed colon and high body of the stomach was found $50 \mathrm{~cm}$ from the upper incisor. The stomach showed normal mucosal findings. A 12 $\mathrm{mm}$ sized residual sessile tumor was found at the interposed colon $38 \mathrm{~cm}$ from the upper incisor. Endoscopic resection was attempted, and piecemeal resection was performed with 2 pieces of the specimen. Gross complete resection was achieved with no visible residual lesion. Argon plasma coag-
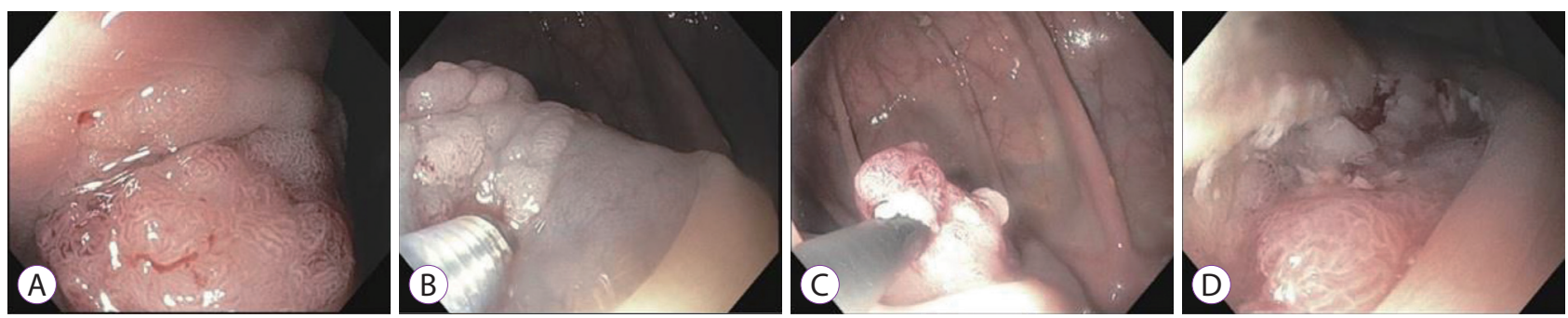

Fig. 1. Esophagogastroduodenoscopy performed at another hospital. (A) Endoscopic image showing a 20-mm sessile polyp in the interposed colon. (B, C) Endoscopic resection was attempted and piecemeal resection was performed. (D) Complete resection was not achieved, and the remnant residual lesion is visible.
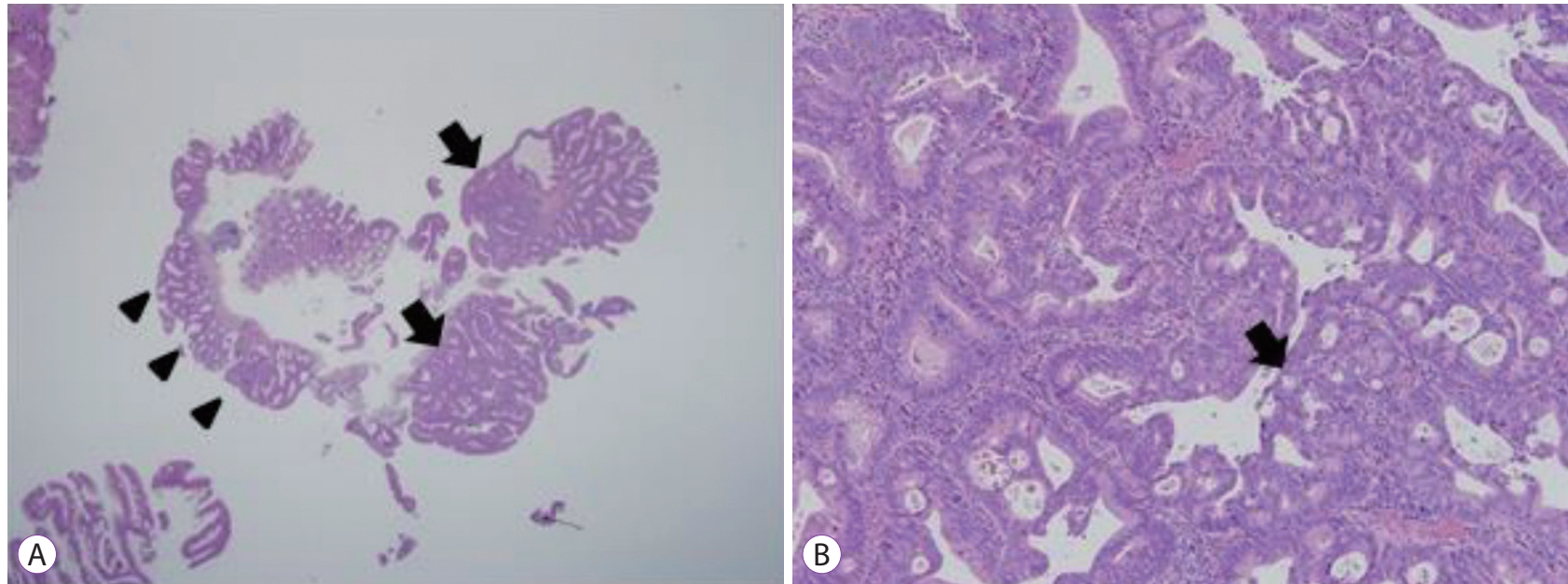

Fig. 2. Histological examination of the endoscopically resected specimen (Hematoxylin and eosin stain). (A) A very low-power scan view image ( $\times 12.5)$ showing simple crypt-like dysplastic glands (arrowheads), a characteristic of villotubular adenoma. The complicated glandular structure suggests adenocarcinomatous changes (arrows). (B) A low-power field view image ( $\times 100)$ showing cribriform formation and back-to-back appearance of a glandular structure with loss of polarity (arrow), suggesting adenocarcinoma. 
ulation (APC) was conducted at the post-resection ulcer base and ulcer edge to eliminate possible residual lesions, thereby, minimizing the risk of local recurrence (Fig. 3). There was no complication during the procedure. Histopathologic examination confirmed tubular adenoma (Fig. 4).

The patient underwent a follow-up esophagogastroduodenoscopy 1 year after the resection, which showed no evidence of recurrence (Fig. 5).

\section{DISCUSSION}

Colon interposition was first introduced in 1911 as a substitute for gastric pull-up surgery for cases in which esophagectomy was required but the stomach could not be used due to prior stomach surgery or combined tumor infiltration in the stomach. Lee et al. analyzed 1,011 Korean cases from June 2000 to June 2013 ; $^{3}$ in their study, 67 patients underwent colon interposition and 944 patients underwent gastric pull-up surgery, and there was no statistically significant difference between the 2 groups regarding postoperative complications such as anastomosis site leakage, stricture, and fistula. The authors thus concluded that colon interposition is a reasonable alternative for gastric pull-up. Similarly, DeMeester reported that morbidity and mortality rates between colon interposition and gastric pull-up were not significantly different in benign esophageal illnesses such as esophageal stricture. ${ }^{4}$ The short-term outcomes after colon interpositions are relatively well-known; however, only few reports have described the long-term follow-up outcomes in patients with colon interposition. This is because colon interposition is usually performed in patients with esophageal cancer, whose long-
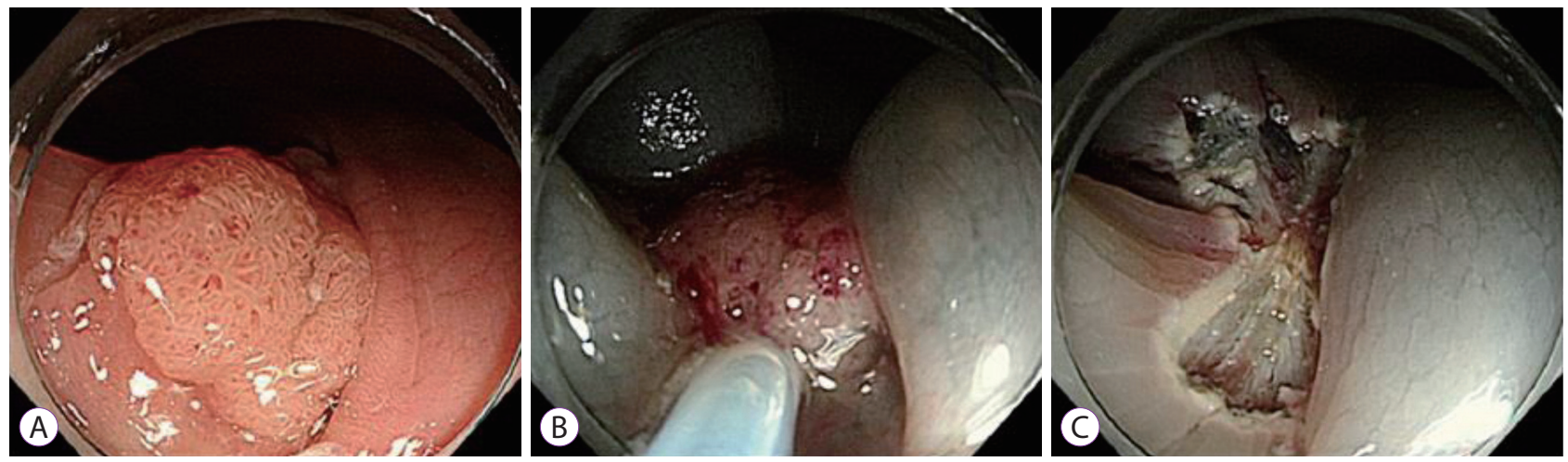

Fig. 3. Repeated endoscopic resection of residual colonic neoplasia at the interposed colon. (A) White light endoscopic image showing a 12-mm residual polyp in the interposed colon. (B, C) Endoscopic mucosal resection was conducted, and the lesion was completely resected by piecemeal fashion in two pieces. Argon plasma coagulation was also performed to eliminate possible microscopic residual lesions.
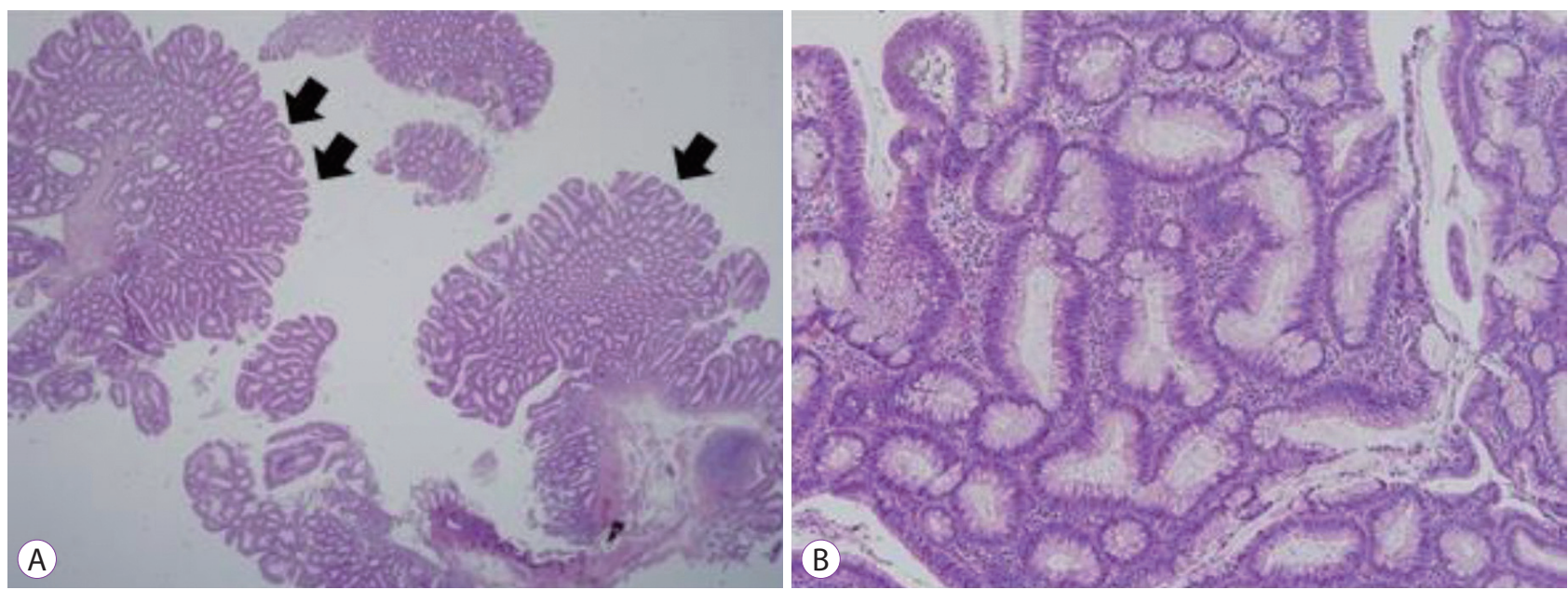

Fig. 4. Histological examination of the specimen resected by repeated endoscopic resection (Hematoxylin and eosin stain). (A) A very low-power scan view ( $\times 12.5)$ showing the typical gross features of the tubular adenoma (arrows). (B) A low-power field view ( $\times 100)$ showing enlarged, hyperchromatic, and elongated nuclei arranged in a stratified configuration along the basement membrane, which is compatible with tubular adenoma. 
term survival rates are low. In contrast, our patient underwent colon interposition due to benign esophageal stricture caused by corrosive esophagitis; therefore, she was able to survive for 40 years after colon interposition before intramucosal cancer developed in the interposed colon.

Intramucosal carcinoma in the colon is limited to the mucosa and is known to have no risk of lymph node metastasis. ${ }^{5}$ Therefore, complete endoscopic resection of intramucosal carcinoma in the colon can achieve a cure without risk of recurrence and/or metastasis. In our current case, complete resection failed at the initial attempt, and repeat endoscopic resection was performed to achieve complete resection of the residual lesion. Although there is no definite consensus on the management of grossly residual intramucosal cancer and/or positive histological resection margin after a grossly complete resection of intramucosal cancer, repeat local eradicative resection may be a plausible option, considering the non-metastatic features of intramucosal colorectal cancer. This suggestion was supported by our previous report on the clinical outcomes of 38 patients with intramucosal colon cancers, which were grossly removed by endoscopic resection but showed positive or uncertain histopathological resection margins. These patients showed neither local recurrence nor distant metastasis for an average of 18.3 months of follow-up without additional intervention. ${ }^{6}$ For a more definite eradication of possible residual lesions, we additionally performed APC after the repeated endoscopic resection. Previous reports showed that APC decreased the risk of local recurrence after piecemeal resection and/or difficult endoscopic resection for recurrent polyps at the fibrotic scar sites. ${ }^{7,8}$ Moreover, the spec- imen of repeated endoscopic resection revealed only benign tubular adenoma with no evidence of malignancy, although the initial endoscopic resection specimen from the other hospital was confirmed as intramucosal carcinoma. This indicates that all carcinoma components were resected in the first endoscopic resection, and only the benign adenoma component remained.

Currently, screening colonoscopy is recommended at 5-10 year intervals after 50 years of age in the average-risk asymptomatic population. ${ }^{9-11}$ Because of the rarity of interposed colons, there is no consensus on a screening endoscopy interval for people with interposed colons. Furthermore, compared to native colons, interposed colons may have differences in intraluminal chemical composition and diversity of intraluminal microbiota because of their different anatomical positions. ${ }^{4}$ Despite such uncertainty, it may be reasonable that the general guidelines for colorectal cancer screening for native colons be applied to interposed colons as well, considering the slowly progressing adenoma-carcinoma sequence during prolonged periods. Another issue regarding endoscopy screening of interposed colons is the necessity of gastric cancer screening. The Korean national cancer screening program for gastric cancer recommends that screening esophagogastroduodenoscopies be carried out every 2 years starting from 40 years of age. Therefore, if we consider the screening program for both the stomach and the colon, an adequate screening strategy for early detection of adenoma and adenocarcinoma in interposed colons should be conducting esophagogastroduodenoscopy every 2 years, because it can observe interposed colons as well as the stomach.
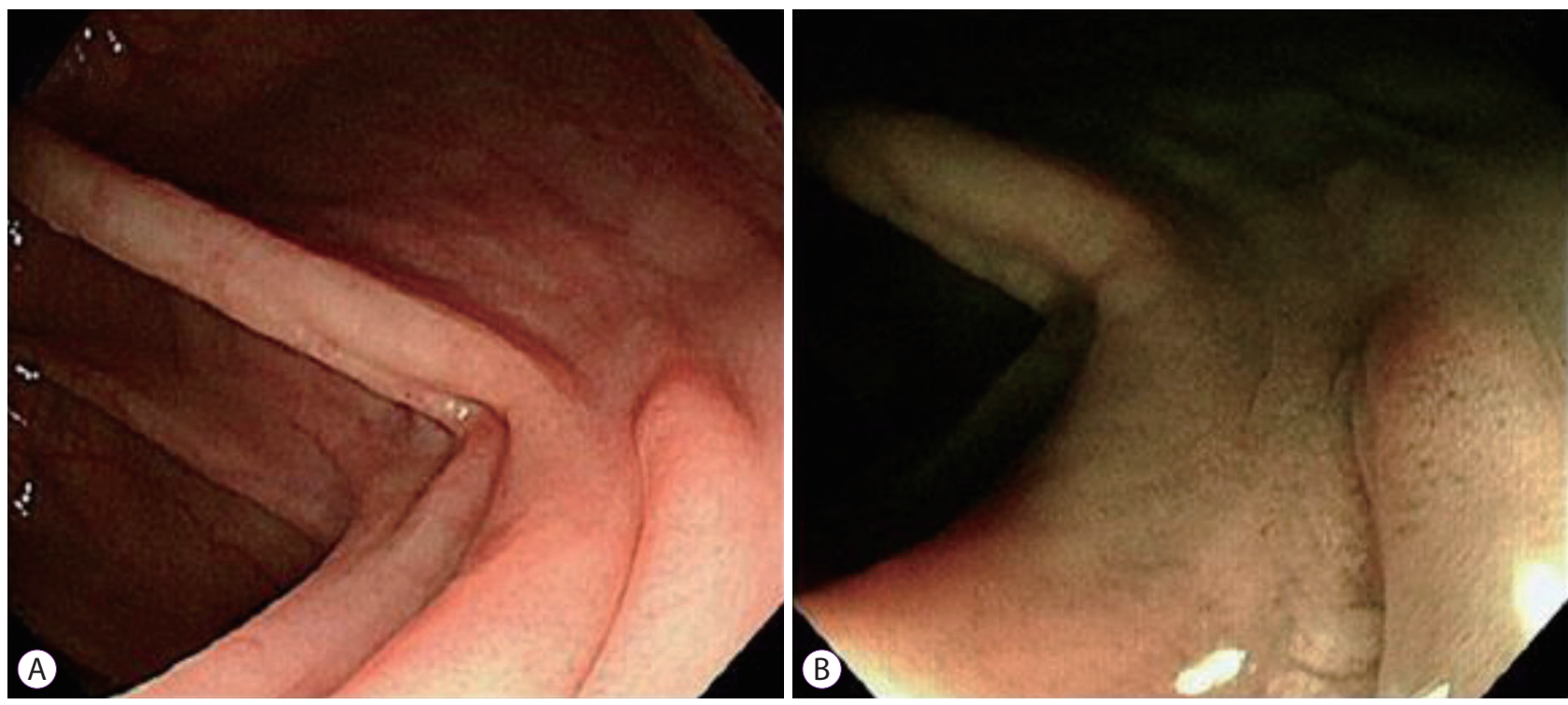

Fig. 5. Follow-up esophagogastroduodenoscopy one year after complete resection of lesion at the interposed colon. A scar was visible at white-light examination (A) and narrow band imaging $(\mathrm{B})$, with no sign of recurrent lesion. 
Reports on adenoma and adenocarcinoma in interposed colons are rare: since the first report in 1968 by Goldsmith et al., 21 cases have been reported. ${ }^{12}$ Nine of the 21 patients underwent esophagectomy with colon interposition due to malignancy, and the remaining had benign esophageal illnesses. In Korea, the first case was reported in 2007 by Hwang et al., which was of an intramucosal cancer in the interposed colon managed by endoscopic resection. ${ }^{13}$ Two more Korean cases were reported afterwards in 2010 and 2012, which were advanced cancers in the interposed colons. ${ }^{14,15}$ Similar to our current case, all reported cases in Korea underwent esophagectomy with colon interposition because of corrosive esophagitis. Supplementary Table 1 summarizes the literature review of adenoma and adenocarcinoma in interposed colons.

In conclusion, we here report a patient who underwent colon interposition surgery due to corrosive esophagitis and was later diagnosed with intramucosal cancer in the interposed colon. The tumor was completely removed by repeated endoscopic resection. Based on our experience and literature review, we suggest that screening endoscopy should be performed for the detection of early neoplasia in the interposed colon; considering the increased possibility of adenoma and adenocarcinoma development, this is especially recommended in patients who survive for a long period after esophagectomy. When considering the necessity of screening esophagogastroduodenoscopy for gastric cancer in the population aged 40 years, interposed colon screening may be performed via esophagogastroduodenoscopy.

\section{Conflicts of Interest}

The authors have no financial conflicts of interest.

\section{REFERENCES}

1. Wilkins EW Jr. Long-segment colon substitution for the esophagus. Ann Surg 1980;192:722-725.

2. Briel JW, Tamhankar AP, Hagen JA, et al. Prevalence and risk factors for ischemia, leak, and stricture of esophageal anastomosis: gastric pull-up versus colon interposition. J Am Coll Surg 2004;198:536-541; discussion 541-542.

3. Lee K, Kim HR, Park SI, Kim DK, Kim YH, Choi SH. Surgical outcome of colon interposition in esophageal cancer surgery: analysis of risk factors for conduit-related morbidity. Thorac Cardiovasc Surg 2018;66:384389.

4. DeMeester SR. Colonic interposition for benign fisease. Oper Tech Thorac Cardiovasc Surg 2006;11:232-249.

5. Fenoglio CM, Kaye GI, Lane N. Distribution of human colonic lymphatics in normal, hyperplastic, and adenomatous tissue. Its relationship to metastasis from small carcinomas in pedunculated adenomas, with two case reports. Gastroenterology 1973;64:51-66.

6. Ko OB, Byeon JS, Kim MJ, et al. Clinical outcomes of colonic mucosal cancers with histologically positive or uncertain resection margin after endoscopic resection. Hepatogastroenterology 2014;61:65-69.

7. Regula J, Wronska E, Polkowski M, et al. Argon plasma coagulation after piecemeal polypectomy of sessile colorectal adenomas: long-term follow-up study. Endoscopy 2003;35:212-218.

8. Tsiamoulos ZP, Bourikas LA, Saunders BP. Endoscopic mucosal ablation: a new argon plasma coagulation/injection technique to assist complete resection of recurrent, fibrotic colon polyps (with video). Gastrointest Endosc 2012;75:400-404.

9. Lee BI, Hong SP, Kim SE, et al. Korean guidelines for colorectal cancer screening and polyp detection. Clin Endosc 2012;45:25-43.

10. Cairns SR, Scholefield JH, Steele RJ, et al. Guidelines for colorectal cancer screening and surveillance in moderate and high risk groups (update from 2002). Gut 2010;59:666-689.

11. Lieberman DA, Rex DK, Winawer SJ, Giardiello FM, Johnson DA, Levin TR. Guidelines for colonoscopy surveillance after screening and polypectomy: a consensus update by the US Multi-Society Task Force on Colorectal Cancer. Gastroenterology 2012;143:844-857.

12. Goldsmith HS, Beattie EJ Jr. Malignant villous tumor in a colon bypass. Ann Surg 1968;167:98-100.

13. Hwang HJ, Song KH, Youn $\mathrm{YH}$, et al. A case of more abundant and dysplastic adenomas in the interposed colon than in the native colon. Yonsei Med J 2007;48:1075-1078.

14. Lee S, Kim ES, Chung WJ, et al. A case of colon cancer developing at the interposed graft for treatment of benign esophageal stricture. Keimyung Medical Journal 2010;29:138-142.

15. Kim ES, Park KS, Cho KB, Kim MJ. Adenocarcinoma occurring at the interposed colon graft for treatment of benign esophageal stricture. Dis Esophagus 2012;25:175. 